\title{
Series expansions for an exact two-electron wave function in terms of Löwdin's renormalized natural orbitals
}

\author{
I. Nagy ${ }^{1,2}$ and I. Aldazabal ${ }^{3,2}$ \\ ${ }^{1}$ Department of Theoretical Physics, Technical University of Budapest, H-1521 Budapest, Hungary \\ ${ }^{2}$ Donostia International Physics Center, P. Manuel de Lardizabal 4, E-20018 San Sebastián, Spain \\ ${ }^{3}$ Centro de Física De Materiales (CSIC-UPV/EHU)-MPC, P. Manuel de Lardizabal 5, E-20018 San Sebastián, Spain
}

(Received 1 February 2012; published 5 March 2012)

\begin{abstract}
In recent developments on the pair density needed to treat the non-Hartree-Fock-like part of interparticle repulsion, the natural orbitals and sign-correct expansion coefficients play a central role. Since, in principle, an infinite number of natural orbitals must be included, the convergence of expectation values due to finite-term approximations is an important issue. Here we discuss quantitatively this convergence problem based on an exactly solvable two-electron model atom, where the Schrödinger wave function for the ground state is expressible in terms of Löwdin's natural orbitals and sign-correct expansion coefficients. Using properly renormalized truncated series expansions for such an exact decomposition, the corresponding expectation values of the Schrödinger Hamiltonian are calculated analytically. A rapid and uniform convergence is found in these expectation values at given values of the coupling in the interparticle repulsion.
\end{abstract}

DOI: 10.1103/PhysRevA.85.034501

PACS number(s): 31.15.ec, 03.65.-w, 03.67.-a, 71.15.Mb

Introduction. The solution of the quantum mechanical many-electron problem is one of the central problems in physics. Besides the density-based mapping [1] to a selfconsistent one-particle problem, the scheme based on the first-order density matrix to construct a total energy functional is a challenging alternative [2,3], since in this scheme one employs an exact expression for the many-body kinetic energy. However, the non-Hartree-Fock-like part of the interparticle repulsion is an unknown functional.

Thus, there is a considerable interest in expressing [4-6] the correlated pair density directly in terms of natural orbitals and their occupation numbers [7]. In principle, an infinite number of natural orbitals must be included, and this results in the important practical question of convergence. Furthermore, even for a two-electron system, where we have a summation over excited closed-shell configurations to expand the exact wave function, one has [6] the crucial phase or sign dilemma. Exactly solvable models should, therefore, provide useful insight into these problems inherent in direct modeling of the pair density.

Results and discussion. The present convergence study is based on Moshinsky's [8] two-electron model Hamiltonian

$$
\begin{aligned}
\hat{H}= & -\frac{\hbar^{2}}{2 m}\left(\frac{d^{2}}{d x_{1}^{2}}+\frac{d^{2}}{d x_{2}^{2}}\right)+\frac{1}{2} m \omega_{0}^{2}\left(x_{1}^{2}+x_{2}^{2}\right) \\
& -\frac{1}{2} m \Lambda \omega_{0}^{2}\left(x_{1}-x_{2}\right)^{2}
\end{aligned}
$$

in one space dimension [9-11]. The coupling, $\Lambda \in[0,0.5]$, measures the strength of a repulsive interparticle interaction. Attraction would correspond to $\Lambda<0$. We start, using Hartree atomic units $\left(e^{2}=\hbar=m=1\right)$, with the exact ground-state wave function

$$
\psi_{\mathrm{ex}}\left(x_{1}, x_{2}\right)=\left(\frac{\omega_{+} \omega_{-}}{\pi^{2}}\right)^{1 / 4} e^{-\frac{1}{2} \Omega_{1}\left(x_{1}^{2}+x_{2}^{2}\right)} e^{-\frac{1}{2} \Omega_{2} x_{1} x_{2}},
$$

where $\Omega_{1}=\left(\omega_{+}+\omega_{-}\right) / 2$ and $\Omega_{2}=\left(\omega_{+}-\omega_{-}\right)$, with $\omega_{+}=$ $\omega_{0}$ and $\omega_{-}=\omega_{0} \sqrt{1-2 \Lambda}$.
In the course of discussing geometric measures of the interparticle interaction based on the above model, we derived [11] the following sign-correct spectral $(s)$ decomposition:

$$
\psi_{s}\left(x_{1}, x_{2}\right)=\sum_{n=0}^{\infty}(-1)^{n}\left(P_{n}\right)^{1 / 2} \phi_{n}\left(x_{1}\right) \phi_{n}\left(x_{2}\right) .
$$

In this equation the coupling-dependent $P_{n}$ numbers [the eigenvalues of the exact one-particle density matrix, derived from the exact $\left.\psi_{\mathrm{ex}}\left(x_{1}, x_{2}\right)\right]$ and the $\phi_{n}(x)$ functions (the eigenfunctions of the same matrix) are given $[9,11,12]$ by the following expressions:

$$
P_{n}=\frac{2 \lambda}{1+\lambda}\left(\frac{1-\lambda}{1+\lambda}\right)^{n}
$$

where $\lambda(\Lambda) \equiv 2 \sqrt{\omega_{+} \omega_{-}} /\left(\omega_{+}+\omega_{-}\right)=2(1-2 \Lambda)^{1 / 4} /(1+$ $\sqrt{1-2 \Lambda})$, and

$$
\phi_{n}(x)=\left(\frac{\sqrt{\bar{\omega}}}{2^{n} n ! \sqrt{\pi}}\right)^{1 / 2} H_{n}(\sqrt{\bar{\omega}} x) e^{-\frac{1}{2} \bar{\omega} x^{2}}
$$

Here, with a $\bar{\omega} \equiv \sqrt{\omega_{+} \omega_{-}}=\omega_{0}(1-2 \Lambda)^{1 / 4}$ shorthand, $H_{n}(x)$ are Hermite polynomials for which $H_{n}(-x)=(-1)^{n} H_{n}(x)$. The polynomials form a complete orthonormal set, and we have a perfect $[13,14]$ overlap between $\psi_{\mathrm{ex}}\left(x_{1}, x_{2}\right)$ and $\psi_{s}\left(x_{1}, x_{2}\right)$ for repulsive interaction. For an attractive coupling, where $\Lambda<0$, the $(-1)^{n}$ weighting factor is not needed in the above infinite sum to get a unit overlap. This difference is codified [11] in the important sign behavior of $\Omega_{2}(\Lambda)$ in the exact $\psi_{\text {ex }}\left(x_{1}, x_{2}\right)$ under a sign change of $\Lambda$.

The exact ground-state energy of $\psi_{\mathrm{ex}}\left(x_{1}, x_{2}\right)$ with the Schrödinger Hamiltonian of Eq. (1) consists of expectation values of kinetic (first term: $\bar{K}_{\text {ex }}$ ), confining potential (second term), and interaction potential (last term) 
energies:

$$
\begin{aligned}
E_{\mathrm{ex}}(\Lambda)= & \frac{1}{4} \omega_{0}(1+\sqrt{1-2 \Lambda}) \\
& +\left(\frac{1}{4} \omega_{0} \frac{1+\sqrt{1-2 \Lambda}}{\sqrt{1-2 \Lambda}}-\frac{1}{2} \omega_{0} \frac{\Lambda}{\sqrt{1-2 \Lambda}}\right) .
\end{aligned}
$$

The sum of the potential energies is equal to the kinetic energy in harmony, for harmonic forces in the present case, with the virial theorem. In our two-particle model system an instability occurs at the $\Lambda \rightarrow 0.5$ critical limit. For $\Lambda>0.5$ the repulsion between the particles is so strong that they cannot both remain in the external potential well [2].

The application of the spectral form in Eq. (3) results [12] in the exact expectation values for the above energy terms. Now, we truncate (tr) the series expansion as

$$
\psi_{s}^{(\operatorname{tr})}\left(x_{1}, x_{2}\right)=\frac{1}{S_{N}} \sum_{n=0}^{N}(-1)^{n}\left(P_{n}\right)^{1 / 2} \phi_{n}\left(x_{1}\right) \phi_{n}\left(x_{2}\right),
$$

where $S_{N} \equiv\left(\sum_{n=0}^{N} P_{n}\right)^{1 / 2}$ in order to have a unit normalization. Notice at this point that for two-electron systems the natural orbital expansion has [7] an additional extremum property. Even a truncated but properly renormalized, i.e., optimized, finite-term expansion has maximum overlap [7,11] with the exact solution of the Schrödinger Hamiltonian. We use in our convergence study below the transparent representation given by Eq. (5).

In practice, we apply from now on a rewritten Schrödinger Hamiltonian

$$
\begin{aligned}
\hat{H}= & -\frac{1}{2}\left(\frac{d^{2}}{d x_{1}^{2}}+\frac{d^{2}}{d x_{2}^{2}}\right)+\frac{1}{2} \Omega^{2}(\Lambda)\left(x_{1}^{2}+x_{2}^{2}\right) \\
& +\Lambda \omega_{0}^{2} x_{1} x_{2},
\end{aligned}
$$

where $\Omega(\Lambda) \equiv \omega_{0} \sqrt{1-\Lambda}$, as in the Hartree-Fock approximation [8], which results in a product state for the interacting two-electron wave function. The important nonseparable part of the original Hamiltonian is transparent. It is, for the present model, out of the Hartree-Fock framework and thus represents Wigner's correlation at the most fundamental, i.e., Hamiltonian, level. Of course, all energy terms (kinetic and potential) contain correlation contributions [cf., Eq. (4)]. Below we apply rules of quantum mechanics for a harmonic oscillator and the expression for the sum $\left(s_{N}\right)$ of a finite geometric $(q<1)$ series,

$$
s_{N}(q)=\sum_{n=0}^{N} q^{n}=\frac{1-q^{N+1}}{1-q} .
$$

With the first and second terms of the rewritten Schrödinger Hamiltonian in Eq. (6), we only have to perform the following type of simple summation,

$$
\sum_{n=0}^{N}\left(n+\frac{1}{2}\right) P_{n}(\Lambda)
$$

to arrive at the average values of the kinetic $\bar{K}^{\text {(tr) }}$ and confining $\bar{C}^{(\text {tr) }}$ energies. By using Srednicki's convenient variable $\xi \equiv(1-\lambda) /(1+\lambda)$, and thus $P_{n}(\xi)=(1-\xi) \xi^{n}$, we obtain

$$
\begin{gathered}
\bar{K}^{(\mathrm{tr})}=\frac{1}{2} \bar{\omega}[1+2 F(N, \xi)], \\
\bar{C}^{(\mathrm{tr})}=\frac{1}{2} \bar{\omega}\left(\frac{\Omega}{\bar{\omega}}\right)^{2}[1+2 F(N, \xi)] .
\end{gathered}
$$

To these expressions the useful [see, at Eq. (11)] abbreviation $F(N, \xi)$ is given by

$$
F(N, \xi)=\frac{\xi}{1-\xi} \frac{1-\xi^{N}[1+N(1-\xi)]}{1-\xi^{N+1}},
$$

and to get the above compact forms we used the fact that $\sum_{n=0}^{N} P_{n}=\left(1-\xi^{N+1}\right)$. This latter sum is, in fact, the square of the overlap integral performed with the exact $\psi_{\text {ex }}$ and a truncated $\psi_{s}^{(\operatorname{tr})}$ wave functions. Notice, furthermore, that the $(-1)^{n}$ weighting factor is not important in the calculation of these expectation values of energies, since $(-1)^{2 n}=1$.

In the calculation of the remaining term, i.e., of the expectation value of the nonseparable term in Eq. (6) with a truncated $\psi_{s}^{(\mathrm{tr})}$, we apply the selection rule for transitions between oscillator states. In such a manner, and for the repulsive $(R)$ interparticle interaction, one has to perform the summation ( $N \geqslant 1$ below) in the following expression:

$$
\begin{aligned}
\bar{R}^{(\mathrm{tr})}= & 2 \Lambda \omega_{0}^{2} \frac{1}{S_{N}} \frac{1}{S_{N+1}} \sum_{n=0}^{N}(-1)^{2 n+1} \\
& \times\left(\frac{n+1}{2 \bar{\omega}}\right) \sqrt{P_{n}} \sqrt{P_{n+1}},
\end{aligned}
$$

which contains, in the big brackets, the square of the matrix element for an allowed transition between oscillator states. After substitutions, we arrive at

$$
\bar{R}^{(\mathrm{tr})}=-\frac{\Lambda \omega_{0}^{2}}{\bar{\omega}} \frac{1}{S_{N} S_{N+1}} \sum_{n=0}^{N}(n+1) \sqrt{\xi}(1-\xi) \xi^{n}
$$

in terms of $\xi(\Lambda)$ and the coupling constant $\Lambda$. By performing the summation, we get

$$
\bar{R}^{(\mathrm{tr})}=-\frac{\Lambda \omega_{0}}{(1-2 \Lambda)^{1 / 4}} \sqrt{\xi}\left(\frac{1-\xi^{N+1}}{1-\xi^{N+2}}\right)^{1 / 2}[1+F(N, \xi)]
$$

which is the last term of the total energy, $\left[\bar{K}^{(\operatorname{tr})}+\bar{C}^{(\mathrm{tr})}+\bar{R}^{(\mathrm{tr})}\right]$, in our consideration. We stress at this point that for $N=0$ the approximate total energy is the sum of $\bar{K}^{(\text {tr) }}$ and $\bar{C}^{(\text {tr) }}$ only, since the last term of Eq. (6) does not give a contribution when $N=0$ in Eq. (5).

Now we illustrate the $N$ dependence of convergence at the $\Lambda=0.499$ value for the inter-particle coupling. To this illustration we use curves for a better visualization, although the calculations were performed, of course, at integer values $(N=1,2,3, \ldots .$.$) of N$. The $\bar{K}^{(\text {tr })} / \bar{K}_{\mathrm{ex}},\left[\bar{C}^{(\mathrm{tr})}+\bar{R}^{(\mathrm{tr})}\right] / \bar{K}_{\mathrm{ex}}$, and the $\left[\bar{K}^{(\mathrm{tr})}+\bar{C}^{(\mathrm{tr})}+\bar{R}^{(\mathrm{tr})}\right] /\left(2 \bar{K}^{(\mathrm{tr})}\right)$ dimensionless ratios are exhibited on Fig. 1. The third ratio (solid curve) shows how the virial theorem is satisfied under truncation in the 


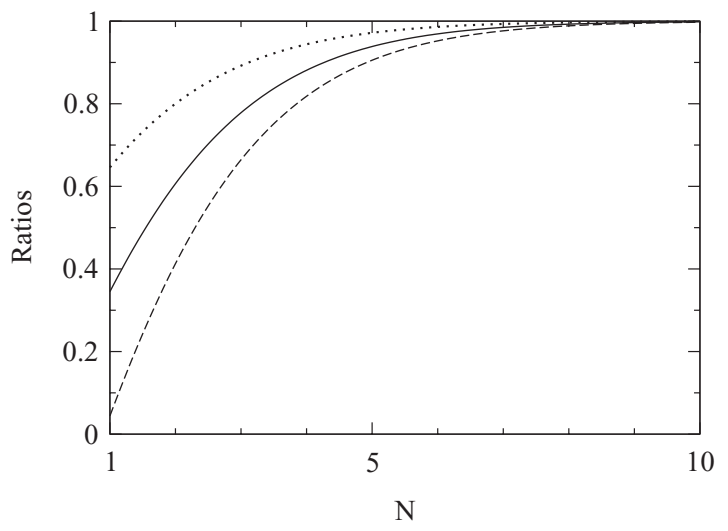

FIG. 1. Different dimensionless ratios, defined by $\bar{K}^{(\mathrm{tr})} / \bar{K}_{\text {ex }}$ (dotted curve), $\left[\bar{C}^{(\mathrm{tr})}+\bar{R}^{(\mathrm{tr})}\right] / \bar{K}_{\mathrm{ex}}$ (dashed curve), and $\left[\bar{K}^{(\mathrm{tr})}+\bar{C}^{(\mathrm{tr})}+\right.$ $\left.\bar{R}^{(\text {tr) }}\right] /\left(2 \bar{K}^{(\text {tr) }}\right)$ (solid curve), as a function of $N$ at $\Lambda=0.499$. The curve representation is used only for visualization. The data are at integer $N$ values.

spectral representation. It is clear from Fig. 1 that the approximate total potential energy (dashed curve) deviates more strongly from the exact limit form (obtained at $N \rightarrow \infty$ ) than the approximate kinetic energy (dotted curve). It is this remarkable difference which signals that it is probably very problematic to describe all, i.e., kinetic and potential, of the correlation effect via, for instance, a functional of the density.

For smaller and smaller $\Lambda$ values the convergence in $N$ is faster and faster. However, very close to the instability limit, i.e., for $\Lambda \rightarrow 0.5$, one can get negative values in small- $N$ expansions. In order to show this we use the notation $\Delta \equiv(1-2 \Lambda) \ll 1$ and expand the above energy terms at $N=1$ for simplicity. Under these prefixed conditions we get the following: $\left(\bar{K}^{\text {(tr) }} / \omega_{0}\right) \simeq \Delta^{1 / 4},\left(\bar{C}^{\text {(tr) }} / \omega_{0}\right) \simeq$ $1 /\left(2 \Delta^{1 / 4}\right)$, and $\left(\bar{R}^{(\text {tr) }} / \omega_{0}\right) \simeq-\sqrt{3 / 2} /\left(2 \Delta^{1 / 4}\right)$. In deriving these, we used the fact that $F(N=1, \xi \rightarrow 1) \simeq(1 / 2)$. Thus, the source of the mentioned negativity resides, in this highly limiting case, in the interplay between $\bar{C}^{\text {(tr) }}$ and $\bar{R}^{\text {(tr) }}$.

We finish our convergence study on energies by a brief discussion of entanglement entropies. The truncated oneparticle density matrix, which is based on the above spectral representation given by Eq. (5), takes the following form:

$$
\gamma^{(\mathrm{tr})}\left(x_{1}, x_{2}, N\right)=\sum_{n=0}^{N} \mathcal{P}_{n} \phi_{n}\left(x_{1}\right) \phi_{n}\left(x_{2}\right),
$$

to which the abbreviation is defined by $\mathcal{P}_{n} \equiv P_{n} /\left(1-\xi^{N+1}\right)$. Of course, $\operatorname{Tr} \gamma^{(\operatorname{tr})}=1$. Taking an operator power of this density matrix, one can describe Rényi's [15,16] entropies as

$$
S_{p}=\frac{1}{1-p} \ln \left[\sum_{n=0}^{N}\left(\mathcal{P}_{n}\right)^{p}\right],
$$

where $p>0$ with $p \neq 1$. At the particularly interesting limit, i.e., at $p \rightarrow \infty$, we get the so-called min- entropy for Moshinsky's interacting two-electron model atom,

$$
S_{\infty}=\ln \left(\frac{1-\xi^{N+1}}{1-\xi}\right),
$$

in terms of $\xi(\Lambda)$. Thus, at $N \gg 1$ we have $S_{\infty}(\Lambda \rightarrow 0)=$ $(\Lambda / 4)^{2}$, which shows a similar scaling $\left(\sim \Lambda^{2}\right)$ as Wigner's correlation energy $E_{\text {corr }}(\Lambda) \equiv\left[\left(\omega_{0} / 2\right) \sqrt{1-\Lambda}-E_{\text {ex }}(\Lambda)\right]$ for small coupling, i.e., when the non-Hartree-Fock-like term in Eq. (6) is small.

Another useful measure of electron-electron interaction is the so-called degree of correlation $(K)$ defined by the following expression:

$$
K^{-1}=\sum_{n=0}^{\infty}\left(P_{n}\right)^{2} .
$$

This has been used earlier [17,18] as a global measure of two-electron correlation. In our case $K=(1 / \lambda)=(1+$ $\sqrt{1-2 \Lambda}) / 2(1-2 \Lambda)^{1 / 4}$, in terms of the coupling $\Lambda$. For small $\Lambda$, i.e., when $K$ is still close (from above) to unity, a renormalized truncated expansion will give (with $\mathcal{P}_{n}$ and $n \leqslant N$, in the above definition) a very reasonable value for this measure.

Of course, different entropies are not expectation values of operators in the Hilbert space. But their approach, at fixed $\Lambda$ and $p$ values, to their limiting values as a function of $N$ can give useful complementary information on entanglement. For instance, the above $S_{\infty}(N)$ also signals, very transparently, a monotonous convergence. Finally, due to the proper renormalization, we get at $N=0$ a zero value for the entropy as in any single-shell representation which would correspond only to an effective one-electron external field.

Summary. There is a considerable interest in expanding the pair density directly in terms of natural orbitals and their occupation numbers in order to calculate the expectation value of the non-Hartree-Fock-like part of interparticle repulsion. However, both the phase dilemma in expansion and the convergence in energy are inherent problems in direct attempts to model this pair density in density-matrix functional theory. In this study we have investigated the convergence problem in the ground-state energy by using properly renormalized finite-term expansions for an exact sign-correct spectral decomposition of the wave function of an exactly solvable two-electron model atom. Based on such expansions, closed analytic expressions are derived for the different constituents of the total energy. The sign of the repulsive energy term $\bar{R}$ reflects the importance of a sign-correct spectral decomposition. The numerical results illustrate the capability of Löwdin's optimized natural orbitals involved in our truncated expansion. The convergence is rapid and uniform.

\section{ACKNOWLEDGMENTS}

One of us (I.N.) is grateful to Professor J. M. Gracia-Bondía and Professor J. Pipek for discussions, and to Professor P. M. Echenique for the warm hospitality at the DIPC. This work was supported partly by the Spanish MICINN (Project No. FIS2010-19609-C02-02). 
[1] W. Kohn, Rev. Mod. Phys. 71, 1253 (1999).

[2] E. R. Davidson, Reduced Density Matrices in Quantum Chemistry (Academic, New York, 1976).

[3] A. J. Coleman and V. I. Yukalov, Reduced Density Matrices, Coulson's Challenge (Springer, Berlin, 2000).

[4] S. Goedecker and C. J. Umrigar, Phys. Rev. Lett. 81, 866 (1998).

[5] M. A. Buijse and E. J. Baerends, Mol. Phys. 100, 401 (2002).

[6] O. Gritsenko, K. Pernal, and E. J. Baerends, J. Chem. Phys. 122, 204102 (2005).

[7] P.-O. Löwdin and H. Shull, Phys. Rev. 101, 1730 (1956).

[8] M. Moshinsky, Am. J. Phys. 36, 52 (1968).

[9] M. Srednicki, Phys. Rev. Lett. 71, 666 (1993).

[10] P. García-González, Phys. Rev. A 79, 062502 (2009).

[11] I. Nagy and I. Aldazabal, Phys. Rev. A 84, 032516 (2011).
[12] Ph. Blanchard, J. Gracia-Bondía, and J. C. Várilly, Int. J. Quantum Chem. 112, 1134 (2012); K. Ebrahimi-Fard and J. Gracia-Bondía, J. Math. Chem. 50, 440 (2012).

[13] F. Riesz and B. Sz.-Nagy, Functional Analysis (Ungar, New York, 1955).

[14] E. H. Lieb and M. Loss, Analysis (American Mathematical Society, Providencia, 2001).

[15] A. Rényi, Probability Theory (North-Holland, Amsterdam, 1970).

[16] M. Ohya and D. Petz, Quantum Entropy and Its Use (Springer, Berlin, 1993).

[17] R. Grobe, K. Rzazewski, and J. H. Eberly, J. Phys. B 27, L503 (1994).

[18] W.-C. Liu, J. H. Eberly, S. L. Haan, and R. Grobe, Phys. Rev. Lett. 83, 520 (1999). 